\title{
On the Improvement of Scaling Laws for Large-Scale MANETs with Network Coding
}

\author{
Chi Zhang, Student Member, IEEE, Xiaoyan Zhu, and Yuguang Fang, Fellow, IEEE
}

\begin{abstract}
This paper investigates the problem of how much benefit network coding can contribute to the network performance in terms of throughput, delay, and storage requirements for mobile ad hoc networks (MANETs), compared to when only replication, storage and forwarding are allowed in relay nodes. We characterize the throughput-delay-storage tradeoffs under different node mobility patterns, i.e., i.i.d. and random walk mobility, with and without network coding. Our results show that when random linear coding instead of replication is used in MANETs, an order improvement on the scaling laws of MANETs can be achieved. Note that previous work showed that network coding could only provide constant improvement on the throughput of static wireless networks. Our work thus differentiates MANETs from static wireless networks by the role network coding plays.
\end{abstract}

Index Terms-Fundamental limits, network coding, scaling laws, throughput-delay-storage tradeoff, mobility, mobile ad hoc networks (MANETs).

\section{INTRODUCTION}

$\mathbf{O}$ NE distinct characteristic of wireless mobile ad hoc networks (MANETs) is that, besides transporting data through multi-hop connected paths between the source and destination, packets can also be delivered through the physical mobility of relay nodes which is called store-carryand-forward paradigm in the literature [1]. Grossglauser and Tse [2] have shown that significant gains in per-node throughput can be obtained by exploiting this paradigm. In particular, they proposed a 2-hop relaying scheme, and showed that it can achieve a constant per-node throughput. The scheme overcomes the throughput bound of $O(1 / \sqrt{n \log n})^{1}$ originally established by Gupta and Kumar [3] for a static wireless network, where $n$ is the number of nodes. Although heavy use of relaying through node mobility allows for higher

Manuscript received August 1, 2008; revised February 20, 2009. This work was partially supported by the U.S. National Science Foundation under grant CNS-0721744, CNS-0716450, CNS-0626881 and DBI-0529012. The work of $\mathrm{X}$. Zhu and Y. Fang was also partially supported by the 111 project under grant B08038 with Xidian University, Xi'an, China.

C. Zhang and Y. Fang are with the Department of Electrical and Computer Engineering, University of Florida, Gainesville, FL 32611 USA. Y. Fang is also a Changjiang Scholar Chair Professor with the National Key Laboratory of Integrated Services Networks, Xidian University, Xi'an, China (e-mail: zhangchi@ufl.edu; fang@ece.ufl.edu).

$X$. Zhu is with the National Key Laboratory of Integrated Services Networks, Xidian University, Xi'an, China (e-mail: xyzhu@mail.xidian.edu.cn).

Digital Object Identifier 10.1109/JSAC.2009.090608.

${ }^{1}$ Throughout the paper we use standard asymptotic notations. For two nonnegative functions $f(\cdot)$ and $g(\cdot)$ : (i) $f(n)=O(g(n))$ means that there exists a constant $c$ and an integer $N$ such that $f(n) \leq c \cdot g(n)$ for $n>N$; (ii) $f(n)=o(g(n))$ means that $\lim _{n \rightarrow \infty} f(n) / g(n)=0$; (iii) $f(n)=\Omega(g(n))$ means that there exists a constant $c$ and an integer $N$ such that $f(n) \geq c \cdot g(n)$ for $n>N$; (iv) $f(n)=\omega(g(n))$ means that $\lim _{n \rightarrow \infty} f(n) / g(n)=\infty$; (v) $f(n)=\Theta(g(n))$ means that $f(n)=O(g(n))$ and $g(n)=O(f(n))$. throughput, it also bears two negative side-effects: increased delay and increased storage requirements. It has been shown in [4], [5] that the 2-hop relaying scheme in [2] yields an extremely large average delay of $\Omega(n)$, whereas the relay buffer size requirement on each node is at least $\Omega(n)$ [6].

Since both throughput and delay are important network performance metrics from the perspective of an application, significant effort in the last few years has been devoted to understand the throughput-delay relationship in MANETs (refer to Section II-A and the references therein) in the networking research community. An interesting work by Neely and Modiano [5] suggested to utilize redundant packets transmission through multiple opportunistic paths (which are composed of multiple opportunistic links) of a MANET to balance the conflicting requirements on throughput and delay. The basic idea is that the time required for a packet to reach the destination (i.e., end-to-end delay) can be reduced by repeatedly transmitting this packet to many relay nodes of the network, and thus improving the chances that some user holding an original or duplicate version of the packet reaches the destination node. Clearly, the cost of this approach is the decreased throughput since duplicate packets waste scarce opportunities of wireless transmissions. In particular, with i.i.d. mobility, it was shown that for per-node throughput $T(n)=O(1)$, the relaying strategies with replication could yield end-to-end delay $D(n)$ scaling as $\Theta(n \cdot T(n))$ [5].

Buffer space of mobile nodes in MANETs is also an important and scarce network resource. Constraints on buffer space/storage reduce the throughput capacity and increase the network delay due to buffer overflow or packet losses. In practice, buffer space is always limited, and its effects on network performance should be quantified. In [6], Herdtner and Chong showed that, given the size of the relay buffer per node, say $b_{n}$, the per-node throughput is at most $O\left(\sqrt{b_{n} / n}\right)$. The relationship of delay with storage and the impact of replication strategies as proposed in [5], however, were not addressed.

Previous studies on the scaling laws of MANETs, as discussed above, are all based on the implicit assumption that each node can only perform traditional operations on packets, such as storage, replication and forwarding. Recently, network coding, first introduced by Ahlswede et al. [7] in 2000, has been widely recognized as a promising primitive operation besides simple replicating and forwarding incoming packets [8]. Using the paradigm of network coding, when a node is scheduled to transmit, it may transmit a "mixed" packet as a result of algebraic operations on its incoming packets to maximize the usefulness of this transmission to 
all receivers in its transmission range. Moreover, when a node receives a new packet and its buffer is full, it will mix the new packet with stored ones in such a way that maximizes the information stored in its buffer. It is worth noting that a particular useful form of network coding called Random Linear Coding (RLC) was proposed in the literature [9], [10] to independently and randomly mix incoming packets at each node with linear operations, which allows the nodes of the network to achieve the optimal performance while operating in a decentralized fashion.

Intuitively, when RLC instead of replication is used to minimize the end-to-end delay, network congestion can be alleviated and the requirement on buffer size can be relaxed. Therefore, a better throughput-delay-storage tradeoff is expected to obtained. Since network coding was not taken into consideration in Grossglauser and Tse's original work [2] and the related work [4]-[6] that followed, an interesting question raised naturally is how much benefit network coding can provide to the network performance of MANETs compared to when only simple replication and forwarding are allowed for relay nodes. Answering this question will help us better understand not only the benefits and limitations of network coding in wireless networks but also the fundamental tradeoffs determining MANET's performance.

In this paper we study the scaling laws governing MANETs. We characterize the throughput-delay-storage tradeoffs with respect to different node mobility patterns. We identify scenarios in which network coding can provide significant improvement on network performance. We also provide insights on when and how information mixing is beneficial and propose algorithms to show that these benefits can be achieved in an effective and decentralized fashion.

The rest of the paper is outlined as follows. Section II provides a review of related work. Section III presents models of MANETs and definitions of network performance metrics. Section IV is included for comparison purposes, which summaries main results on throughput-delay-storage tradeoffs for MANETs using replication strategies, instead of network coding. Section V and Section VI investigate throughputdelay-storage tradeoffs when network coding is used. Finally, Section VII concludes the paper.

\section{BACKGROUND AND RELATED WORK}

\section{A. Scaling Laws of MANETs without Network Coding}

In general, there are two ways to trade throughput for delay in the literature. Kleinrock and Silvester [11] may be the first to find that delay of multi-hop routing can be reduced by increasing the transmission radius of each relay node, at the expense of reducing the number of simultaneous successful transmissions the network can support, which leads to a lower throughput. Similar transmission radius scaling techniques have appeared in [4], [12]-[18]. Another approach, which improves delay via redundant packet transfers is considered in [5], [19]. In this paper, we follow this approach, adopting replication strategy and comparing it with network coding for the following reasons:

- First of all, the assumption that transmission ranges can scale with $n$, the number of nodes, is impractical for large-scale MANETs. To obtain the scaling law of MANETs, we usually require $n$ tending to infinity, which is equivalent to assuming $\sqrt{A_{n}} \rightarrow \infty$ for extended network model, where $A_{n}$ is the area of the network (cf. Section III-A). In general, wireless device is power limited, rendering it impossible to require the transmission range reaching the order of $\sqrt{A_{n}}$.

- Secondly, in this paper we are interested in examining pure gains introduced by network coding in MANETs. Replication strategies can be replaced by network coding, which provides a good chance for comparison. Transmission radius scaling techniques, however, are orthogonal to network coding, and should be studied separately.

\section{B. Scaling Laws of Wireless Networks with Network Coding}

Scaling laws governing wireless networks with network coding have only been investigated in the limited scenarios in the literature recently. The delay gains and reliability benefit (measured in the reduced number of transmissions) of network coding in unreliable wireless networks were characterized in [20], [21] and [22], respectively. However, these results are for one multicast session with one-hop transmission or stable network topology. For multiple unicast scenario, Liu et al. [23], [24] and Keshavarz-Haddadt et al. [25] showed that for static wireless networks, network coding and broadcasting at most provide a constant-factored improvement in the throughput, compared to Gupta and Kumar's $\Theta(1 / \sqrt{n \log n})$ per-node throughput [3]. However in this paper, our results show that, network coding can provide significant improvement on network performance when mobility is utilized, which is impossible in static wireless networks [23]-[25]. We believe it reveals the intrinsic difference between MANETs and static wireless networks.

Very recently, Ying et al. [18] proposed joint codingscheduling schemes to improve the throughput-delay tradeoffs for MANETs using rateless codes (e.g. Raptor codes). Note that their work [18] is different from ours in that: (1) They also considered adjusting transmission range (or cell size) to achieve a better tradeoff. Therefore it is difficult to say that the gain in their scheme is purely due to the coding. (2) The results in [20] are restricted to cases when delay $D(n)$ is both $\omega(\sqrt[3]{n})$ and $o(n)$. In our paper, we design the schemes with $D(n)=\Theta(\log n)$ and $D(n)=\Theta(n)$.

\section{MANET MODELS AND DEFINITIONS}

\section{A. Network Models}

Random network model for MANETs: Consider an ad hoc network where $n$ nodes are initially uniformly distributed at random in a square torus of area $A_{n}$. We consider a multiple ( $n$ ) unicast scenario in which each node $i \in\{1,2, \cdots, n\}$ is a source node for one unicast session, and a destination node for another unicast session. Suppose that the source node $i$ has data intended for destination node $d(i)$. We assume that each source node has an infinite stream of packets to send to its destination. The source-destination (S-D) association does not change with time, although the nodes themselves move.

Mobility models: The torus is divided into $m=\Theta(n)$ square cells of area $A_{n} / m$ each, resulting in a two-dimensional $\sqrt{m} \times$ 


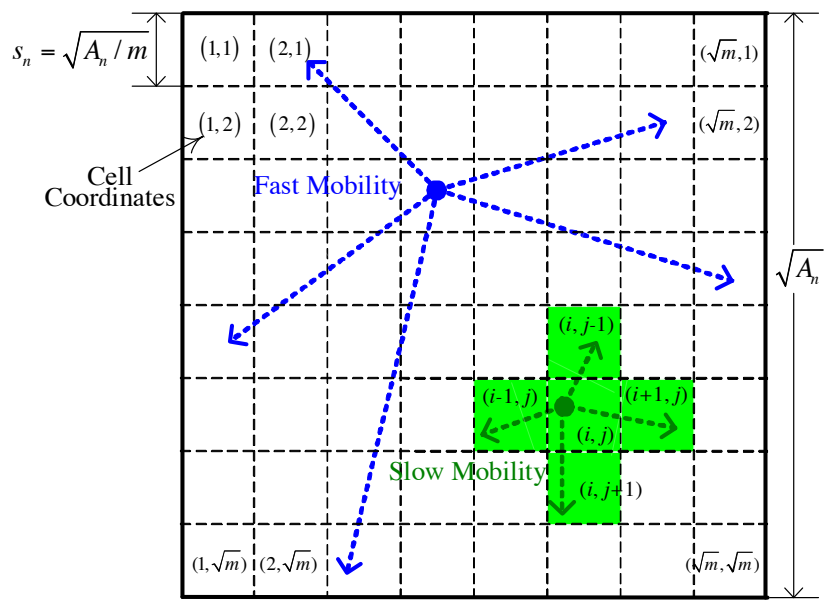

Fig. 1. Fast and slow mobility models for MANETs.

$\sqrt{m}$ discrete torus, see Fig. 1 for an illustration. The initial position of each node is equally likely to be any of the $m$ possible cells independent of others. We further assume the time is slotted and we study the following mobility models in this paper:

- Two-dimensional i.i.d. mobility model (fast mobility model): At each time slot, nodes randomly choose a new cell location independently and identically (i.i.d.) distributed over all cells in the network. This model captures the situation when mobile user moves so quickly that its position is almost independent from time to time. With this assumption, the network topology dramatically changes in every time slot, so that the network behavior cannot be predicted and fixed routing algorithms cannot be used. This mobility model is also used in [5], [12], [14], [17], [18].

- Two-dimensional random walk model (slow mobility model): Let a node be in cell $(i, j) \in\{1, \cdots \sqrt{m}\}^{2}$ at time slot $t$, then, at time slot $t+1$, the node is equally likely to be in the same cell $(i, j)$ or any of the four adjacent cells $\{(i-1, j),(i+1, j),(i, j-1),(i, j+1)\}$, where addition and subtraction are modulo $\sqrt{m}$. So each node in fact independently performs a simple random walk on the two-dimensional $\sqrt{m} \times \sqrt{m}$ discrete torus. Note that this model implicitly sets an upper-bound on the velocity of mobile nodes as $\sqrt{2 A_{n} / m}$. Therefore, it is a suitable model for capturing real motion of nodes with slow mobility. Similar mobility model is also adopted in [4], [15]-[18].

Model for successful transmission: For characterizing the condition for a successful transmission, we adopt the Protocol model as defined in [3]. We assume that all nodes use a common range $r_{c}$ for their transmissions, and a transmission from node $i$ to node $j$ is successful if and only if $d_{i j} \leq r_{c}$ and $d_{k j} \geq(1+\Delta) r_{c}$ for any other simultaneous transmitter, say node $k$. Here, $d_{i j}$ is the distance between nodes $i$ and $j$, and $\Delta$ is a positive constant independent of $n$.

Concurrently transmitting cells: Now we define the transmission range and schedule. We choose $r_{n}$ in such a way that any node in all cells can always directly transmit to



Fig. 2. Cell transmission scheduling. Here is an illustration of the cells being divided into $K^{2}$ groups for the case of $K=4$, i.e., 16 groups. All the blue cells which are in group 1 transmit in the same timeslot. In the next timeslot all the cells in group 2 transmit and so on.

any other node in the same cell using the smallest common range of transmission. Obviously, $r_{c}=\sqrt{2} s_{n}=\sqrt{2 A_{n} / m}=$ $\Theta\left(\sqrt{A_{n} / n}\right)$.

Time is slotted for packetized transmission. We assume only $O(1)$ packets can be transmitted per cell per timeslot, i.e., our analysis is explicitly based on constant packet size model (see [16] and Section II-A for detailed discussion).

We say that a cell is active in a timeslot if any of its nodes transmits in that timeslot, and a cell $(i, j)$ interferes with another cell $(k, l)$ if a transmission by a node in cell $(i, j)$ can affect the success of a simultaneous transmission by a node in cell $(k, l)$. Consider in Protocol model, two interferencefree cells vertically or horizontally $K-1$ cells apart. We know that in order to guarantee the successful transmissions in these two cells, we need $(K-1) \cdot s_{n} \geq(1+\Delta) \cdot r_{n}$ (refer to nodes' positions at the top right of Fig. 2 for an illustration). Let $K=\lceil 1+(1+\Delta) \sqrt{2}\rceil$, we divide all cells into $K^{2}$ groups. All cells belonging to the same group are at vertical or horizontal distance of exactly some multiples of $K$, and can transmit simultaneously as depicted in Fig. 2. Now, we can design a finite length time-division scheduling scheme of $K^{2}$ timeslots, in which each cell group is assigned one slot to transmit. Fig. 2 gives an example of such cell scheduling with $K=4$. Based on the above discussion, we have the following Proposition.

Proposition 1: Under the Protocol model, there exists an interference-free schedule such that each cell becomes active regularly once in $K^{2}$ timeslots and it does not interfere with any other simultaneously transmitting cells. Here $K$ depends only on $\Delta$, and is independent of $n$.

Extended network model: We are particularly interested in asymptotic properties of MANETs, which hold with high probability $^{2}$ for large-scale MANETs. Therefore, we need often take limits as $n \rightarrow \infty$. When the region area $A_{n}$ is fixed, it corresponds to a dense network model [3], [26], since the density of the network $d=n / A_{n}$ also tends to

\footnotetext{
${ }^{2}$ We say that an event occurs with high probability (w.h.p.) if its probability tends to 1 as $n \rightarrow \infty$.
} 
infinity as $n$. Another widely used model is the extended network model [27], [28], in which both the number of nodes and the area of the region $A_{n}$ go to infinity while $d$ is kept constant. Both models are widely used in the literature and we will focus on the latter one. In the extended network model, $A_{n}=n / d=\Theta(n)$, and correspondingly $r_{c}=\Theta\left(\sqrt{A_{n} / n}\right)=\Theta(1)$, which is independent of $n$. This is more practical, since power constraint on wireless devices does not change when more nodes are added to the network. We note that, however, results obtained in this paper can be easily extended to dense network model.

\section{B. Network Performance Metrics}

Definition of throughput: A throughput $\lambda>0$ is said to be feasible/achievable if every node can send at a rate of $\lambda$ bits per second to its chosen destination. We denote by $T(n)$, the maximum feasible throughput w.h.p. Given a scheme $\Pi$, let $M_{\Pi}(i, t)$ be the number of packets from source node $i$ that destination node $d(i)$ receives in $t$ timeslots under scheme $\Pi$, for $1 \leq i \leq n$. Note that this could be a random quantity for a given realization of the network. Define the long term throughput of S-D pair $i$, denoted by $\lambda_{\Pi}^{i}(n)$, to be

$$
\lambda_{\Pi}^{i}(n)=\liminf _{t \rightarrow \infty} \frac{1}{t} M_{\Pi}(i, t) .
$$

Scheme $\Pi$ is said to have throughput $T_{\Pi}(n)$ if

$$
\lim _{n \rightarrow \infty} \mathbb{P}\left(\lambda_{\Pi}^{i}(n) \geq T_{\Pi}(n) \text { for all } i\right)=1 .
$$

We allow randomness in schemes and as a result random quantities above are in the joint probability space including both the random network of size $n$ and the scheme $\Pi$. Note that when network coding is utilized in scheme $\Pi, M_{\Pi}(i, t)$ is the number of successfully decoded packets received by the destination $d(i)$ of S-D pair $i$ in $t$ timeslots under scheme $\Pi$.

Definition of delay: The delay of a packet is the time it takes the packet to reach the destination after it leaves the source. We do not take queueing delay at the source into account, since our interest is in the network delay. Let $D_{\Pi}^{i}(j)$ denote the delay of packet $j$ of S-D pair $i$ under scheme $\Pi$, then the sample mean of delay (over packets that reach their destinations) for S-D pair $i$ is

$$
\bar{D}_{\Pi}^{i}=\limsup _{k \rightarrow \infty} \frac{1}{k} \sum_{j=1}^{k} D_{\Pi}^{i}(j) .
$$

The average delay over all S-D pairs for a particular realization of the random network is then $\bar{D}_{\Pi}=\frac{1}{n} \sum_{i=1}^{n} \bar{D}_{\Pi}^{i}$. The delay for a scheme $\Pi$ is the expectation of the average delay over all S-D pairs and all random network configurations, i.e.,

$$
D_{\Pi}(n)=\mathbb{E}\left[\bar{D}_{\Pi}\right]=\frac{1}{n} \sum_{i=1}^{n} \mathbb{E}\left[\bar{D}_{\Pi}^{i}\right] .
$$

Note that when network coding is utilized, we consider the delay of getting original packets. When an original packet $m_{i}$ belongs to the generation $M$, the delay of $m_{i}$ under scheme $\Pi$ is the time from the first packet belonging to $M$ departs the source to the original packet $m_{i}$ has been decoded at the destination.

\section{Throughrut-Delay-Storage Tradeoffs WITHOUT NETWORK CODING}

In this section, we give a brief overview of the redundancybased schemes as presented in [5] and establish the throughput-delay-storage tradeoffs in MANETs without network coding.

\section{A. Throughput-Delay Tradeoffs with Infinite Buffer Spaces}

We first describe three relay schemes with different redundancy proposed in [5] from a unified point of view.

\section{Three Redundancy-Based Schemes Proposed in [5]:}

We can control the transmission redundancy of each packet with two methods: the number of hops each packet will take from source to destination, and the total number of copies (replicas) of each original packet in the network. The three schemes, namely, 2-hop relay without replicas, 2-hop relay with $k_{1}$ replicas, and multi-hop relay with $k_{2}$ replicas represent different combinations of the two methods.

Each scheme has two parts: (1) scheduling of active cells; (2) scheduling of transmission in an active cell.

The three schemes have the same cell scheduling policy (Part (1)) as follows:

- Each cell becomes active once in every $K^{2}$ timeslots as discussed in Proposition 1.

- In an active cell, transmission is always between two nodes within the same cell.

In every active cell with at least two nodes, intra-cell transmission scheduling (Part (2)) is needed.

- For 2-hop relay schemes, each packet at most takes two hops from source to destination. At every timeslot, a transmitter-receiver (T-R) pair is selected randomly from all node pairs in each active cell. With probability $1 / 2$, the chosen $\mathrm{T}-\mathrm{R}$ pair will act in source-to-relay $(S \rightarrow R)$ mode or relay-to-destination $(R \rightarrow D)$ mode. The difference is that, for 2-hop relay without replicas, packets are not duplicated and are held by at most one node (source or relay) at any timeslot, while for 2-hop relay with $k_{1}$ replicas, in $S \rightarrow R$ mode, the source will send $k_{1}$ replicas to distinct nodes as relays, and in $R \rightarrow D$ mode, the receiver tells its transmitter which packet it is looking for before the transmission begins (using handshake), to guarantee that the receiver always gets new useful packets when it acts as a destination node.

- Multi-hop relay with $k_{2}$ replicas is just another type of flooding scheme, which transmits $k_{2}$ replicas of each packet, and places no constrains on the number of hops. It assumes that each packet is stamped with the timeslot $t$ in which it first leaves the source. At every timeslot in each active cell, of all packets that are contained in at least one node of the cell and that have never been received by any other node in the same cell, the packet that has the smallest timestamp $t$ will be selected to send to all nodes in the cell (i.e., the local oldest policy for packet transmission scheduling).

Theorem 1: Assuming infinite buffer space at each node, throughput-delay tradeoffs achieved by the three redundancybased schemes proposed in [5] for MANETs under fast 
mobility model can be summarized in the following table.

\begin{tabular}{|c|l|l|}
\hline scheme & throughput & delay \\
\hline 2-hop relay without replicas & $\Theta(1)$ & $\Theta(n)$ \\
2-hop relay with $k_{1}$ replicas & $\Theta(1 / \sqrt{n})$ & $\Theta(\sqrt{n})$ \\
multi-hop relay with $k_{2}$ replicas & $\Theta\left(\frac{1}{n \log n}\right)$ & $\Theta(\log n)$ \\
\hline
\end{tabular}

Note that the performance above is achieved with $k_{1}=$ $\Theta(\sqrt{n})$ and $k_{2}=\Theta(\log n)$, respectively.

Remark 1: The handshake used in $R \rightarrow D$ mode for 2hop relay with $k_{1}$ redundancy, and the local oldest policy for packet transmission scheduling used in multi-hop relay with $k_{2}$ redundancy, are adopted to reduce unnecessary transmission redundancy and delay, which is critical for achieving optimal throughput-delay tradeoffs. Note that these two techniques can also be replaced by a naive technique called Random Message Selection (RMS) proposed in [29] and [30], which selects the packet to be transmitted randomly without considering the receiver's preference. Therefore, it is possible for RMS to schedule the transmission of a packet already in the receiver's buffer, causing decreased throughput and increased delay. For example, it has been shown that the delay for RMS is $\Theta(n \log n)$, much larger than that of the schemes described here. The benefit of RMS is that it simplifies the algorithm running in each node, and saves the communication overhead introduced by complex techniques. For extended network model used in this paper, node density in each cell is small, i.e., $d=\Theta(1)$. The overhead and complexity introduced by the two redundancy-based schemes can be ignored. However, when the node density in each cell is high, e.g., in dense network model, optimal redundancy-based schemes are impractical, and RMS is the only choice, where the gain of using network coding will be amplified by a factor of $\Theta(\log n)$ as shown in [29] and [30].

Theorem 2: Assuming infinite buffer space at each node, throughput-delay tradeoffs achieved by the three redundancybased schemes proposed in [5] for MANETs under slow mobility model can be summarized in the following table.

\begin{tabular}{|c|l|l|}
\hline scheme & throughput & delay \\
\hline 2-hop relay without replicas & $\Theta(1)$ & $\Theta(n \log n)$ \\
2-hop relay with $k_{1}$ replicas & $\Theta\left(\frac{1}{\sqrt{n} \log n}\right)$ & $\Theta(\sqrt{n} \log n)$ \\
multi-hop relay with $k_{2}$ replicas & $\Theta\left(\frac{1}{n \sqrt{n}}\right)$ & $\Theta(\sqrt{n})$ \\
\hline
\end{tabular}

Note that the performance above is achieved with $k_{1}=$ $\Theta(\sqrt{n} \log n)$ and $k_{2}=\Theta(\sqrt{n})$, respectively.

\section{B. Throughput-Storage Tradeoffs}

In this subsection, we analyze the impacts of finite or limited buffer size on the scaling properties of MANETs. Note that [6] only provides a loose upper bound on throughput given buffer size $b_{n}$ (cf. Section II-A). Our results presented here provide a tighter bound than the previous ones in [6].

Theorem 3: Assume each node has a buffer space of $b_{n}$ packets, the throughput is upper-bounded by $O\left(\frac{b_{n}}{b_{n}+n}\right)$, and this bound is achievable using 2-hop relay without replicas.

Due to space constraints, we omit the proof here.
Remark 2: For a given S-D pair, the average packet queue size in the network is the product of per node throughput and average packet life time (i.e., the average delay) using Little's law. Therefore, the average number of packets for a given pair (i.e., the number of buffers required to support a given S-D pair's flow) is determined by the throughput-delay product. From this observation, the average buffer requirements for supporting optimal throughput-delay tradeoffs in Theorem 1 are $O(n), O(1)$, and $O(1 / n)$, respectively. Therefore, we can conclude that the higher the redundancy in a scheme, the lower the requirement on the buffer size. At a first glance, this result conflicts with our intuition since excessive replication will tend to waste buffer space. However, the results presented in Section IV-A show that replication reduces both throughput and delay because we trade throughput for delay improvement. As a result, the throughput-delay product decreases as well.

\section{Throughrut-Delay-Storage Tradeoffs With Network CODING: SCHEMES AND REsults}

We first review RLC used in our network coding based schemes. This bears exactly the same setup as in [29]. Then we describe the schemes developed for analyzing tradeoffs in MANETs with network coding, and identify scenarios in which RLC improves network performance of MANETs significantly.

\section{A. Network Coding Operation}

Random linear coding (RLC) is applied to a finite set of $k$ original packets (i.e., $M=\left\{m_{1}, m_{2}, \cdots, m_{k}\right\}$ ), that is called a generation. Each packet is viewed as an $r$-dimensional vector over a finite field, $\mathbb{F}_{q}$ of size $q$, i.e., $m_{i} \in \mathbb{F}_{q}^{r}$, $i=1,2, \cdots, k$. If the packet size is $m$ bits, this can be done by viewing each packet as an $r=\left\lceil\mathrm{m} / \log _{2}(q)\right\rceil$-dimensional vector over $\mathbb{F}_{q}$ (instead of viewing each packet as an $m$ dimensional vector over binary field). Typically, $\mathbb{F}_{2^{8}}$ (i.e., $\mathbb{F}_{256}$ ) is used. All the additions and multiplications in the following description are assumed to be over $\mathbb{F}_{q}$. We assume that all the $k$ packets in $M$ are linearly independent. During the execution of a RLC based relay scheme, the destination node of $M$ collects linear combinations of the packets in $M$. Once there are $k$ independent linear combinations at a node, it can recover all the original packets in $M$ successfully.

Now, consider a certain timeslot $t$. Let $S_{v}(t)$ and $S_{u}(t)$ denote the set of all the coded packets (each coded packet is a linear combination of the packets in $M$ ) at node $v$ and $u$, respectively, at the beginning of the timeslot $t$. More precisely, if coded packet $f_{l} \in S_{v}(t)$, where $l=1,2, \cdots,\left|S_{v}(t)\right|$, then $f_{l} \in \mathbb{F}_{q}^{r}$ has the form $f_{l}=\sum_{i=1}^{k} \alpha_{l i} \cdot m_{i}, \alpha_{l i} \in \mathbb{F}_{q}$. The scheme ensures that $a_{l i}$ 's are known to node $v$ by appending each packet $f_{l}$ with a "code vector", which will be explained a little later. Let $S_{v}(t)^{-}$and $S_{u}(t)^{-}$denote the subspaces spanned by the coded packets in $S_{v}(t)$ and $S_{u}(t)$, respectively. If $S_{v}(t)^{-} \nsubseteq S_{u}(t)^{-}$, we say node $v$ has useful information about $M$ for $u$. In timeslot $t$, if node $v$ is scheduled by the scheme to transmit a packet related to $M$ to node $u, v$ first checks if it has useful information for $u$. If so, $v$ transmits a 
"random" coded packet with payload $f_{n e w} \in \mathbb{F}_{q}^{r}$ to $u$, where $f_{\text {new }}=\sum_{f_{l} \in S_{v}(t)} \beta_{l} \cdot f_{l}, \beta_{l} \in \mathbb{F}_{q}$ and $\mathbb{P}\left(\beta_{l}=\beta\right)=\frac{1}{q}, \forall \beta \in \mathbb{F}_{q}$.

It is easy to check that $f_{n e w}$ is still a linear combination of the $k$ original packets, and can be written as $f_{\text {new }}=\sum_{i=1}^{k} \theta_{i} \cdot m_{i}$ where $\theta_{i}=\sum_{f_{l} \in S_{v}(t)} \beta_{l} \cdot \alpha_{l i} \in \mathbb{F}_{q}$. For decoding purposes, the vector $\left(\theta_{1}, \theta_{2}, \cdots, \theta_{k}\right) \in \mathbb{F}_{q}^{r}$, called code vector, will be appended to $f_{n e w}$, and sent as overhead. This overhead clearly requires a padding of additional $k \log _{2}(q)$ bits. If the packet size $m \gg \log _{2}(q)$, which would be the case under our constant packet size model, then the overhead required by the RLC based scheme can be ignored in our analysis. ${ }^{3}$ We say that $v$ sends an innovative coded packet $f_{n e w}$ to $u$, if $f_{\text {new }}$ can increase the dimension of the subspace $S_{u}(t)^{-}$, i.e., $\operatorname{dim}\left(S_{u}(t)^{-}\right)$. Note that $\operatorname{dim}\left(S_{u}(t)^{-}\right) \leq k$ in general and if $\operatorname{dim}\left(S_{u}(t)^{-}\right)=k$, node $u$ can recover all the $k$ original packets at once. We now recall the following key result about RLC, which says that $f_{\text {new }}$ will be an innovative coded packet for $u$ with probability no less than $1-\frac{1}{q}$.

Proposition 2: (Lemma 2.1 in [29]) Let $S_{u}(t)^{+}=S_{u}(t) \cup$ $\left\{f_{n e w}\right\}$ be the subspace spanned by the code vectors in $u$ at the end of timeslot $t$, i.e., after receiving a coded packet $f_{\text {new }}$ from $v$ according to the RLC based scheme described as above. Then,

$\mathbb{P}\left(\operatorname{dim}\left(S_{u}(t)^{+}\right)>\operatorname{dim}\left(S_{u}(t)^{-}\right) \mid S_{v}(t)^{-} \nsubseteq S_{u}(t)^{-}\right) \geq 1-\frac{1}{q}$

\section{B. RLC-Based Relay Schemes}

In this subsection, we describe RLC-based relay schemes with different routing strategies, which will be used later to exploit throughput-delay tradeoffs in MANETs.

We first introduce the concept of big generation. In what follows, when we say that the source node groups $k=$ $\omega(\log n)$ original packets into one big generation, we in fact separate these $k$ packets into $k / \Theta(\log n)$ generations, each with $\Theta(\log n)$ packets. When the destination node tries to decode one original packet, it first needs to collect $\Theta(k)$ coded packets from the big generation (with $\Theta(\log n)$ coded packets from each generation). Therefore the overhead introduced by RLC is ignorable in our analysis (cf. footnote 3 ).

\section{Schemes 1: 2-hop Relay with RLC}

(1) $k$ original packets in each source node will be grouped into one (big) generation. Each source will send $m=(1+\epsilon) k$ coded packets for each (big) generation, where $\epsilon$ is a constant. (2) Coded packets for each generation will have the same timestamp $t_{p}$. The value of $t_{p}$ is the time the first coded packet of that generation leaves the source. All coded packets of a generation will be deleted from the relay buffer at the timeslot $t$ if $t-t_{p}>t h_{p}$, where the threshold $t h_{p}$ depends on $D(n)$ of the scheme and will be sufficiently larger than $D(n)$.

\footnotetext{
${ }^{3}$ More precisely, the constant packet size model for original packets means that the packet size scales as $\Theta(\log n)$ bits, since it needs to carry the ID of the destination node with $\Theta(\log n)$ bits. For a fair comparison, we require that $k=O(\log n)$ for the coded packets throughout the paper. Therefore the overhead introduced by the code vector will not change the order of our results on $T(n)$ and $D(n)$ for RLC-based schemes.
}

(3) Each cell becomes active once in every $K^{2}$ timeslots as discussed in Proposition 1. In an active cell, transmission is always between nodes within the same cell.

(4) For an active cell with at least two nodes, a random transmitter-receiver pair is selected, with uniform probability over all possible node pairs in the cell. With probability $1 / 2$, the transmitter is scheduled to operate in either "Source-toRelay" or "Relay-to-Destination" mode, described as follows:

- Source-to-Relay Mode: The transmitter sends a coded packet of its current generation, and does so upon every transmission opportunity while it is in source-to-relay mode until $m$ coded packets have been delivered to distinct nodes. If all other nodes in the cell already have one coded packet for that generation, the source will begin to transmit coded packets from the next generation. Every node stores a single packet per S-D pair per generation. When the node receives a new packet, a relay linearly combines the incoming packet with the stored one, and replaces the stored packet with the result. Note that the nodes operate in broadcast mode, i.e., every node will hear every transmission in its range, and update the packet storage as described above.

- Relay-to-Destination Mode: If the designated transmitter has a coded packet in its relay buffer for the destination node, and the rank of coded packets of that generation in the receiver is smaller than $k$, the coded packet is transmitted to the designated receiver.

Remark 3: Since $m>k$, we need a mechanism to stop unnecessary relay of coded packets of a generation when it is already decoded in the destination. Here we use a proactive stopping mechanism, i.e., the timestamp of each generation, since we can bound the delay of the scheme. In the analysis part presented later, we will show that $k=\Theta(n)$, and $D(n)$ for this scheme is also $\Theta(n)$ for fast and slow mobility models. Therefore, $t h_{p}$ should be larger than $\Theta(n)$. More complicated reactive stopping mechanisms (cf. [31] and the references therein) can be adopted to enhance the efficiency of the scheme in practice. However, we follow the simplest design for analytical tractability of the scheme.

\section{Schemes 2: Multi-hop Relay with RLC}

(1) $k$ original packets in each source node will be grouped into one (big) generation. Each source will send $m=(1+\epsilon) k$ coded packets for each generation, where $\epsilon$ is a constant. Two timestamps for each generation are used. One is called the generating time $t_{g}$, based on the time for $k$ original packets to be grouped into a generation in the source. Another is called transmission time $t_{p}$, based on the time the first coded packet of that generation is transmitted by the source.

(2) Each cell becomes active once in every $K^{2}$ timeslots as discussed in Proposition 1. In an active cell, transmission is always between nodes within the same cell.

(3) For an active cell with at least two nodes, perform the following: among all packets contained in at least one node of the cell and which have useful information for some other node in the same cell, choose the packet with the smallest generating time $t_{g}$. If there are ties, choose the packet from the S-D pair $i$ which maximizes $\left(t_{g}+i\right) \bmod n$. Transmit this packet to all other nodes in the cell. If the selected packet is in 
the source, then the source will transmit the linear combination of its $k$ original packets of the same generation, instead of a particular packet belonging to that generation.

(4) Every node stores a single packet per S-D pair per generation. When the node receives a new packet, a relay linearly combines the incoming packet with the stored one, and replaces the stored packet with the result.

(5) All coded packets of a generation will be deleted from the relay buffer at the timeslot $t$ if $t-t_{p}>t h_{p}$, where the threshold $t h_{p}$ depends on $D(n)$ of the scheme and should be sufficiently larger than $D(n)$.

Remark 4: The generating timestamp $t_{g}$ is used to construct a flooding scheme for one particular S-D pair where all $n$ S-D pairs are active and share the network resource. It is easy to see that the packets from the oldest generation that has not been delivered to all nodes will dominate the transmissions over the whole network very quickly. The long-term fairness between all S-D pairs is guaranteed since in the case of ties, packets from S-D pair $i$ are given top priority in every $n$ timeslots. Also note that, since at one particular timeslot, only one generation from one S-D pair dominates the whole network, the number of packets each relay needs to store in step (4) is 1 , i.e., just for one generation w.h.p. Another timestamp $t_{p}$ used here has the same functionality as the previous scheme. The threshold $t h_{p}$ should be larger than $D(n)$, scaling as $\Theta(\log n)$ and $\Theta(\sqrt{n})$, respectively, for fast and slow mobility models.

\section{Main Results about RLC-Based Schemes}

In this subsection, we summarize the performance of the above schemes under different mobility models. Here, we focus on the intuition and explanation of these results. Proofs of theses results will be given in the next section.

Theorem 4: When 2-hop relay with RLC scheme is used and $k=\Theta(n)$, we have $T(n)=\Theta(1)$ and $D(n)=\Theta(n)$ for fast and slow mobility models.

Remark 5: Compare to Theorems 1 and 2, it is easy to see that, RLC provides delay improvement $\Theta(\log n)$ under slow mobility model. No gain is found under fast mobility model. It is not surprising, since 2-hop relay with RLC scheme is used to replace 2-hop relay without replicas, and we know that in the latter, there is no duplicated packets in order to maximize the throughput. Thus we cannot expect any gains when network coding is used. The gain $\Theta(\log n)$ of delay under slow mobility model comes from the lower information propagation speed, and the mixing of packets increase this speed by guaranteeing that every packet the destination received from relay nodes will contribute some information for the decoding of the packet from the same generation. For fast mobility model, this benefit vanishes since the information propagation speed is high enough, and the delay for waiting $k$ coded packets for decoding dominates the whole delay.

Theorem 5: When multi-hop relay with RLC scheme is used, under fast mobility model with $k=\Theta(\log n)$, we have $T(n)=\Theta(1 / n)$ and $D(n)=\Theta(\log n)$. Under slow mobility model with $k=\Theta(\sqrt{n})$, we have $T(n)=\Theta(1 / n)$ and $D(n)=\Theta(\sqrt{n})$.

Remark 6: Under fast and slow mobility models, multihop RLC-based schemes always provide significant gains compared to flooding schemes. We can see that the RLC-based scheme can achieve minimal delay, with an improved delayconstrained throughput. The intuition is that, when flooding is used, there exist enough opportunities to enhance performance by replacing replicas with more intelligent coding.

Fig. 3 compares timetables of 2-hop and multi-hop RLCbased relay schemes. It can be found that in 2-hop relay schemes, multiple sessions operate in a parallel fashion, while in multi-hop relay schemes, they operate in a sequential fashion. Therefore, at each timeslot, for 2-hop relay schemes, traffic pattern is still multiple unicasts. For multiple unicasts, we seldom find gains from network coding. While for multihop relay schemes, at each timeslot, traffic pattern looks more like one broadcast session, where gains from network coding are naturally expected.

Remark 7: Also notice that, multi-hop relay schemes can be divided into multiple phases, and in each phase, relaying for one generation from one S-D pair will dominate the network, which is in fact a type of information flooding in this phase (refer to Fig. 3(b) for an illustration). The result is that in each phase, packets from one generation will be broadcasted to the whole network, and if the other $n-1$ nodes are receivers, they can all decode the original packets in that generation at the end of that phase. So it guarantees that multi-hop relay with RLC coding can support all-to-all traffic pattern ( $n$ broadcast sessions) with the same performance. Note that this also means that the same network performance can be achieved for any $n$ multicast sessions (since receivers in this case are just a subset of receivers in the broadcast case). From Theorem 5, we can easily obtain the following corollary on the performance of multiple broadcasts and multicasts with network coding.

Corollary 1: For all-to-all communications or any multicasts with $n$ sources, when multi-hop relay with RLC scheme is used, under fast mobility model with $k=\Theta(\log n)$, we have $T(n)=\Theta(1 / n)$ and $D(n)=\Theta(\log n)$. Under slow mobility model with $k=\Theta(\sqrt{n})$, we have $T(n)=\Theta(1 / n)$ and $D(n)=\Theta(\sqrt{n})$.

In [30], Fragouli et al. designed an RLC-based scheme based on results from [29]. For all-to-all communications, they showed that their scheme achieves $T(n)=\Theta(1 / n)$ and $D(n)=\Theta(n)$ under fast mobility model. Obviously, their scheme obtained the same throughput as ours at the cost of much larger delay. The basic idea of their scheme is that, $k$ packets from $k$ different sources will be grouped into one generation, and the relaying scheme is essentially the same as ours. The comparison here raises an interesting question - why in our RLC-based schemes we only mix packets from the same source? The reasons are the following: first of all, as shown in the above comparison, even for all-to-all communication scenarios, mixing packets from different sources is not a good choice. Second, for multiple unicast scenarios, we mix packets from different sources and these packets have different destinations. When one destination decodes a packet designated for another destination, this packet is in fact a duplicate at the first destination which will reduce the throughput. In our multi-hop relay with RLC, we also introduce redundancy for the same reason. However, the redundancy here is explicitly designed for decreasing the delay. While for the former case, it is purely a waste of network resource in multiple unicast 


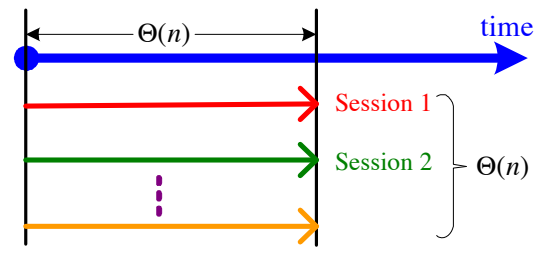

(a) 2-hop relay with RLC coding

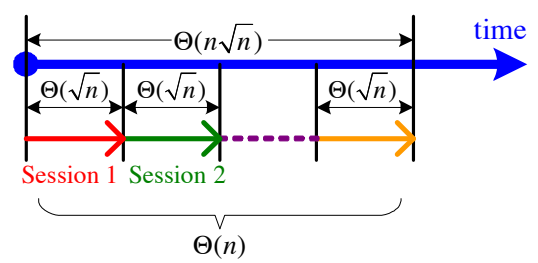

(b) Multi-hop relay with RLC coding

Fig. 3. Timetables for different RLC-based schemes under slow mobility model.

scenarios. Finally, grouping packets from different sources requires coordinations. We are not sure about the cost for performing this coordination task, and we are interested in designing fully decentralized schemes, in which the operations from different nodes should be decoupled as much as possible.

From the above discussion, we can ascertain that for 2hop relay with RLC, have one packet per S-D pair in any relay node is enough for obtaining the performance proposed in Theorem 4, while one packet per node is enough for multi-hop relay with RLC. We can conclude that the storage requirements for these two schemes are exactly $n$ and 1 , respectively, for each node. Compared to the results on the storage requirements for redundancy-based schemes in Theorem 3, we obtain the following Corollary:

Corollary 2: RLC cannot provide improvement on storage requirements better than a constant factor.

It is easy to check that the throughput-storage tradeoffs obtained by RLC-based schemes in this paper follow the same principle provided in Theorem 3 .

\section{Throughput-Delay-Storage Tradeoffs With NETWORK CODING: ANALYSIS}

\section{A. Preliminaries}

The following lemma is useful in delay analysis, since it confirms that the effect of (intra-cell) transmission scheduling only contributes to a constant factor, which can be ignored in asymptotic analysis. Therefore, the time for two desired nodes to meet will dominate the delay of the scheme.

Lemma 1: In the schemes mentioned above, every node will be scheduled to transmit or receive a packet in each timeslot with a constant, non-vanishing probability that is independent of $n$.

Proof: This result can be obtained from Proposition 1. It only depends on the steady state node location distribution. Note that fast and slow mobility models have the same node location distributions in the steady state. Therefore, this result applies to both mobility models.

To facilitate the theoretical analysis, we need first investigate two critical delays for fast and slow mobility models: minimal delays for 2-hop relays and for flooding. Here, 2-hop relay represents any scheme with controlled redundancy on the number of hops (in the 2-hop relay case, the number of hops for each packet is 2, and other schemes with constant hop constrains will yield the critical delays on the same order of $n$ ), and flooding represents all schemes that remove this constraint totally.

Consider the following situation: initially, only one node's color is red, which we call the source. All other nodes are blue. Whenever a source node meets a blue node ${ }^{4}$, the latter is colored red. The time for $\Theta(n)$ nodes to become red is called minimal 2-hop delay. If we change the rule slightly: whenever a red node meets a blue node, the latter is colored red, then the corresponding time is named as minimal flooding delay. Obviously, these two critical delays reflect the intrinsic properties of how mobility will facilitate information propagation. These two quantities are scheme-independent, i.e., they hold for any scheme with or without replicas and with or without network coding.

We first consider two critical delays for fast mobility model.

Lemma 2: The minimal 2-hop delay and the minimal flooding delay under fast mobility model are $\Theta(n)$ and $\Theta(\log n)$, respectively.

Proof: (a) For the minimal flooding delay, Lemma 3 in [5] already established that the number of timeslots required for the source node to send packets to at least $n / 2$ nodes is $\Theta(\log n)$.

(b) For the minimal 2-hop delay, we use the following ballinto-bin argument: For a given source node, we have $(n-2)$ possible relay nodes (i.e., $(n-2)$ bins). At each timeslot, the source node select 1 relay node and send a distinct coded packet to it (i.e., dropping one ball in a selected bin) with a constant probability (cf. Lemma 1). Note that we ignore this probability in the following analysis, since it will not change the order of our result. Repeat this $m=\Theta(n)$ times (i.e., $m$ balls into $(n-2)$ bins), and denote the number of distinct relay nodes as $N$ (i.e., the number of non-empty bins). Here, we need to prove that $N=\Theta(n)$ for some $m=\varepsilon n$ where $\varepsilon$ is a constant. Obviously, every bin is empty with probability $\exp (-m / n)$ independently when $n$ goes to infinity. We assume these $n$ bins as a $\sqrt{n} \times \sqrt{n}$ grid. Based on site percolation results [27], when $1-\exp (-m / n) \geq 0.60$, the number of non-empty bins is on the order of $\Theta(n)$. Therefore, we can always find a constant $\varepsilon$, such that $N=\Theta(n)$ with $m=\varepsilon n$.

Next, we present the results for slow mobility model.

Lemma 3: The minimal 2-hop delay under slow mobility model is $\Theta(n)$.

Proof: Here we need to show that after $m=\Theta(n)$ timeslots, there are $N=\Theta(n)$ distinct red nodes in the network. Based on Lemma 1, the source node $u$ (the first

\footnotetext{
${ }^{4}$ We say that node $u$ meets node $v$ if and only if nodes $u$ and $v$ are in the same cell and scheduled as the sender-receiver pair. Recall that for random walk mobility model discussed in the literature, meeting is defined as two nodes being in the same cell. In this paper we do not distinguish these two kinds of meets since in our model the total number of cells is $\Theta(n)$. Therefore, based on Lemma 1, the (intra-cell) transmission scheduling only contributes to a constant factor, which can be ignored in the following asymptotic analysis.
} 
red node) will be scheduled to transmit $m^{\prime}=\Theta(n)$ times in $m$ timeslots. Obviously $N \leq m^{\prime}$ since we cannot guarantee that every time when node $u$ is scheduled to be the sender, it will transmit a packet to a node it never met before.

Let $N(u, v, m)$ denote the number of times that node $u$ meets $v$ within $m$ timeslots. Under random walk mobility model, the joint position of two nodes due to independent random walks can be viewed as a difference random walk relative to the position of one node. Then the inter-meeting times are just the inter-visit times of cell $(1,1)$ for the difference random walk on a $\sqrt{n} \times \sqrt{n}$ torus. Let $\tau$ be the random variable representing the inter-meeting time defined as above, El Gamal et al. [15] prove that:

Lemma 4: $\mathbb{E}[\tau]=n$ and $\operatorname{Var}[\tau]=\Theta\left(n^{2} \log n\right)$.

Therefore, $N(u, v, m)=m / \tau=\Theta(n / \tau)$ (cf. footnote 4). Recall that for a random variable $X$, we have $\operatorname{Var}[f(X)] \approx$ $\left(f^{\prime}(\mathbb{E}[X])\right)^{2} \operatorname{Var}[X]$. Therefore, for $m=\Theta(n)$, we have $\mathbb{E}[N(u, v, m)]=\Theta(1)$ and $\operatorname{Var}[N(u, v, m)]=\Theta(\log n)$.

Let $V$ be the set of distinct nodes that source node $u$ meets in $m$ timeslots. For two distinct nodes $v_{1}$ and $v_{2} \in V, N\left(u, v_{1}, m\right)$ and $N\left(u, v_{2}, m\right)$ are two independent random variables with the same distribution. Note that $\mathbb{E}\left[\sum_{v \in V} N(u, v, m)\right]=m$. Because $\mathbb{E}\left[\sum_{v \in V} N(u, v, m)\right]=E[N] \cdot E[N(u, v, m)]$, we obtain that $\mathbb{E}[N]=\Theta(n)$. For two random variables $X$ and $Y$, if $\operatorname{Var}[X]$ exists, we have the general formula for variance decomposition as the following: $\operatorname{Var}[X]=\operatorname{Var}[\mathbb{E}[X \mid Y]]+\mathbb{E}[\operatorname{Var}[X \mid Y]]$. Therefore,

$$
\begin{aligned}
\operatorname{Var}\left[\sum_{v \in V} N(u, v, m)\right]= & \mathbb{E}\left[\operatorname{Var}\left[\sum_{v \in V} N(u, v, m) \mid N\right]\right] \\
& +\operatorname{Var}\left[\mathbb{E}\left[\sum_{v \in V} N(u, v, m) \mid N\right]\right] \\
= & \mathbb{E}[N] \cdot \operatorname{Var}[N(u, v, m)] \\
& +\operatorname{Var}[N] \cdot \mathbb{E}[N(u, v, m)] \\
= & \Theta(n) \Theta(\log n)+\operatorname{Var}[N] \Theta(1) .
\end{aligned}
$$

Also note that $|V| \leq m$, therefore

$$
\operatorname{Var}\left[\sum_{v \in V} N(u, v, m)\right] \leq m \cdot \operatorname{Var}[N(u, v, m)]=\Theta(n \log n) .
$$

From above discussions on $\operatorname{Var}\left[\sum_{v \in V} N(u, v, m)\right]$, we obtain that $\operatorname{Var}[N]=O(n \log n)$.

By Chebyshev inequality, for any $0<\kappa<1$,

$$
\mathbb{P}\{N \leq(1-\kappa) \mathbb{E}[N]\} \leq \frac{\operatorname{Var}[N]}{\kappa^{2}(\mathbb{E}[N])^{2}}=O\left(\frac{\log n}{n}\right) \rightarrow 0,
$$

which means that $N=\Theta(n)$ w.h.p.

Lemma 5: The minimal flooding delay under slow mobility model is $\Theta(\sqrt{n})$.

Proof: (a) We first show that the minimal delay is $\Omega(\sqrt{n})$. From random walk model, node speed is upper bounded by $\sqrt{2 A_{n} / m}=O(1)$ and the transmission range $r_{c}=\Theta(1)$. Therefore, information propagation speed will be no larger than $\Theta(1)$ per timeslot. It can be shown that the distance between the initial positions of S-D pair is $\Omega\left(\sqrt{A_{n}}\right)=\Omega(\sqrt{n})$ w.h.p. [32]. Hence, the expected delay is at least $\Omega(\sqrt{n})$ timeslots. (b) We then show that the $\Theta(\sqrt{n})$ delay is achievable using flooding. We cite the following important result about rumor spreading on torus: Theorem 3 in [33] states that following the flooding rule mentioned in Section VI-A, at timeslot $t$, there exists a sub-torus of size $\sqrt{t} \times \sqrt{t}$, where for each cell in this sub-torus, there exists at least one red node. Therefore, in $\Theta(\sqrt{n})$ timeslots, we can cover the whole torus of size $\sqrt{n} \times \sqrt{n}$ w.h.p.

\section{B. Performance of 2-Hop Relay with RLC}

In this subsection we prove Theorem 4 for Scheme 1.

\section{Delay of Scheme 1 under Slow Mobility Model:}

We consider a decoupled version of Scheme 1, which consists of three decoupled phases in time axis:

(1) The source node successfully transmits $m$ coded packets of its current (big) generation to $m$ distinct relay nodes. It takes $N_{1}$ timeslots, and obviously $N_{1} \geq m=\Theta(n)$.

(2) These $m$ relay nodes take independent random walks which takes $N_{2}$ timeslots. After this phase, $m$ relay nodes will be uniformly distributed in the torus.

(3) The destination node collects $k=\Theta(n)$ coded packets from the network. It takes $N_{3}$ timeslots and obviously $N_{3} \geq$ $k=\Theta(n)$.

Obviously $D(n)=N_{1}+N_{2}+N_{3}$, and in what follows we will prove $D(n)=\Theta(n)$ by showing that $N_{1}=N_{2}=N_{3}=$ $\Theta(n)$. Note that instead of collecting coded packets as soon as possible, here the destination node begin to collect packets after $N_{1}+N_{2}$ timeslots. Obviously this strategy is not as efficient as Scheme 1. However, its decoupling nature leads to analytical tractability. We will show that the inefficiency introduced by this decoupling strategy will not change the order of the delay of Scheme 1.

Phase 1: From Lemma 3, we directly obtain that $N_{1}=$ $\Theta(n)$.

Phase 2: From [34], we know that the mixing time of a simple random walk on a $\sqrt{n} \times \sqrt{n}$ torus is also $\Theta(n)$. Therefore, there exist a constant $\varepsilon$ such that after $N_{2}=\varepsilon n=\Theta(n)$ timeslots, these $m$ nodes with coded packets are uniformly distributed in the torus w.h.p. which means that each node in the network has coded packets with a constant probability.

Phase 3: Given that $m$ relay nodes are uniformly distributed over the torus, here we need to prove that after $N_{3}=\Theta(n)$ timeslots, the source node can collect $k$ distinct coded packets. Recall that Lemma 1 shows that the destination node will be scheduled as the receiver with a non-vanishing probability $p_{s}$ in each timeslot, which is independent of $n$. Let $N_{3}^{\prime}$ denote the number of timeslots required by the destination node to meet $m$ distinct relay nodes, we have that $N_{3}^{\prime}=\Theta\left(N_{3}\right)$. Therefore, we only need to prove that $N_{3}^{\prime}=\Theta(n)$. Recall the proof in Lemma 3, we know that after $N_{3}^{\prime}=\Theta\left(N_{3}\right)$ timeslots, the source node will meet $k=\Theta(n)$ distinct relay nodes and obtain $k$ distinct coded packets w.h.p.

\section{Throughput of Scheme 1 under Slow Mobility Model:}

According to above discussion, there are $\Theta(n)$ nodes that can have successful transmission simultaneously at any timeslot. Consider the transmission of packets from relays to destinations. Let $A(i, t)$ be the number of coded packets received by the destination $d(i)$ in timeslot $t$ and $A(t)=\sum_{i=1}^{n} A(i, t)$ 
be the total number of coded packets received in timeslot $t$, we have $\mathbb{E}[A(t)]=\Theta(n)$. Note that the mobile random network is an irreducible finite-state Markov chain and $A(t)$ is a bounded non-negative function of the state of this Markov chain at time $t$. Therefore by the ergodicity of such a Markov chain,

$$
\lim _{T \rightarrow \infty} \frac{1}{t} \sum_{t=1}^{T} A(t)=\mathbb{E}[A(t)]=\Theta(n) .
$$

Thus the total rate at which coded packets are transmitted from relays to destinations is $\Theta(n)$. From the symmetry of the nodes and the randomness of the scheme it follows that each of the $n$ destinations receives at rate of $\Theta(1)$ of coded packets. Since each coded packet contains information of $m / n=\Theta(1)$ original packets, the throughput is still $\Theta(1)$ in original packets.

\section{Delay of Scheme 1 under Fast Mobility Model:}

We consider a decoupled version of Scheme 1, which consists of two decoupled phases in time axis:

(1) The source node successfully transmits $m$ coded packets of its current (big) generation to $m$ distinct relay nodes. It takes $N_{1}$ timeslots, and obviously $N_{1} \geq m=\Theta(n)$.

(2) The destination node collects $k$ coded packets from the network. It takes $N_{2}$ timeslots and obviously $N_{2} \geq k$.

Phase 1: From Lemma 2, we directly obtain that $N_{1}=$ $\Theta(n)$.

Phase 2: For the destination node to meet $k=\Theta(n)$ distinct relay nodes, it is a standard coupon collector problem which requires that $N_{2}=\Theta(n \log n)$. When RLC is used, it is proved in [29], [30] that the destination node only needs to collect $\Theta(n)$ coded packets, which requires that $N_{2}=\Theta(n)$.

Therefore the total delay $D(n)=N_{1}+N_{2}=\Theta(n)$.

\section{Throughput of Scheme 1 under Fast Mobility Model:}

In [35], [36], it is shown that the capacity region depends only on the steady state node location distribution. Note that, for both i.i.d. and random walk mobility models, in steady state nodes are independently and uniformly distributed over the torus. Therefore, a given scheme will achieve the same throughput under these two different mobility models. Recall the result proved under random walk mobility model, we have $T(n)=\Theta(1)$ under i.i.d. mobility model also.

\section{Performance of Multi-Hop Relay with RLC}

In this subsection, we prove Theorem 5 for Scheme 2.

\section{Delay of Scheme 2 under Fast Mobility Model:}

We consider a decoupled version of Scheme 2, which consists of two decoupled phases in time axis:

(1) The source node successfully transmits $m=\Theta(\log n)$ distinct coded packets of its current (big) generation to relay nodes. According to Lemma 1, it only takes $N_{1}=\Theta(\log n)$ time-slots on average. After this phase, according to the flooding scheme analyzed in Lemma 2 w.h.p. each node will have at least one coded packets. Obviously, some relay nodes may have the same coded packets. However, based on the property of mobility pattern (i.i.d.), the information contained in $k=\Theta(\log n)$ distinct coded packets is uniformly distributed over the torus.
(2) According to Proposition 2, the destination node collects $k$ coded packets and recovers the original $k$ packets within $\Theta\left(N_{2}\right)=\Theta(\log n)$ timeslots.

\section{Throughput of Scheme 2 under Fast Mobility Model:}

Note that in order to enable $\Theta(\log n)$ delay in the general case where $n$ S-D pairs are active and share the network resources, we apply a flooding protocol in Scheme 2 in which the oldest generation that has not been delivered to all nodes is selected to dominate network resources. Scheme 2 is "fair" in that in case of ties, session $i$ packets are given top priority every $n$ timeslots. Since we get $k=\Theta(\log n)$ original packets in $\Theta(k)$ timeslots, the throughput for the phase when the transmissions of this $\mathrm{S}-\mathrm{D}$ pair dominate the network is $\Theta(1)$. For fairness embedded in the scheme, this situation happens once for $\Theta(1 / n)$ phases. Therefore, the long-term throughput is $T(n)=\Theta(1 / n)$.

\section{Delay of Scheme 2 under Slow Mobility Model:}

We consider a decoupled version of Scheme 2, which consists of three decoupled phases in time axis:

(1) The source node successfully transmits $k=\Theta(\sqrt{n})$ distinct coded packets of its current generation to relay nodes. According to Lemma 1, it only takes $N_{1}=\Theta(\sqrt{n})$ time-slots on average.

(2) Relay nodes with coded packets take independent random walks and perform packet "mixing" according to RLC rules. Note that, unlike i.i.d. mobility model, which will change the node position in a global-scale in each timeslot, it is not so obvious that whether after $N_{2}=\Theta(\sqrt{n})$ timeslots, the information contained in $k=\Theta(\sqrt{n})$ distinct coded packets is uniformly distributed over the torus under random walk mobility model. Recall that it has been proved in Lemma 5 that based on our flooding scheme and RLC rules, w.h.p. after $\Theta(\sqrt{n})$ timeslots, every given packet can spread over the whole torus. Therefore, we can find a constant $\varepsilon$ such that after $N_{2}=\varepsilon n$ timeslots, the information contained in $k=\Theta(\sqrt{n})$ distinct coded packets is uniformly distributed.

(3) According to Proposition 2, the destination node collects $k$ coded packets and recovers the original $k$ packets within $\Theta\left(N_{2}\right)=\Theta(\sqrt{n})$ timeslots.

\section{Throughput of Scheme 2 under Slow Mobility Model:}

The proof is similar to the proof under fast mobility model. The only difference is replacing $k=\Theta(\log n)$ in the above proof with $k=\Theta(\sqrt{n})$ for slow mobility model.

\section{CONCLUDING REMARKS}

In this paper, we characterize the throughput-delay-storage tradeoffs in mobile ad hoc networks (MANETs) with network coding, and compares with the scenarios where only replication and forwarding are allowed in each node. The schemes/protocols achieving those tradeoffs in an effective and decentralized way are proposed. The scenarios in which network coding provides significant improvement on network performance are identified under different node mobility patterns. The insights on when and how information mixing is beneficial for MANETs with multiple unicast and multicast sessions are provided. 


\section{REFERENCES}

[1] S. Farrell and V. Cahill, Delay- and Disruption-Tolerant Networking. Boston, MA: Artech House, 2006.

[2] M. Grossglauser and M. D. Tse, "Mobility increases the capacity of ad hoc wireless networks," in Proc. IEEE INFOCOM 2001, Anchorage, Alaska, April 2001.

[3] P. Gupta and P. R. Kumar, "The capacity of wireless networks," IEEE Trans. Inform. Theory, vol. 46, no. 2, pp. 388-404, March 2000.

[4] A. E. Gamal, J. Mammen, B. Prabhakar, and D. Shah, "Throughputdelay trade-off in wireless networks," in Proc. IEEE INFOCOM 2004, Hong Kong, China, Mar. 2004.

[5] M. Neely and E. Modiano, "Capacity and delay tradeoffs for ad-hoc mobile networks," IEEE Trans. Inform. Theory, vol. 51, no. 6, pp. 19171937, June 2005.

[6] J. Herdtner and E. Chong, "Throughput-storage tradeoff in ad hoc networks," in Proc. of IEEE INFOCOM 2005, Miami, FL, Mar. 2005.

[7] R. Ahlswede, N. Cai, S. R. Li, and R. W. Yeung, "Network information flow," IEEE Trans. Inform. Theory, vol. 46, no. 4, pp. 1204-1216, July 2000.

[8] C. Fragouli and E. Soljanin, Network coding applications, Foundations and Trends in Networking. Boston, MA: Now Publishers Inc., 2007.

[9] S. Jaggi, P. A. Chou, and K. Jain, "Low complxity optimal algebraic multicast codes," in Proc. IEEE International Symposium on Information Theory, Yokohama, Japan, June-July 2003.

[10] T. Ho, R. Koetter, M. Medard, D. R. Karger, and M. Effros, "The benefits of coding over routing in a randomized setting," in Proc. IEEE International Symposium on Information Theory, Yokohama, Japan, June-July 2003.

[11] L. Kleinrock and J. A. Silvester, "Optimum transmission radii in packet radio networks or why six is a magic number," in Proc. National Telecommunications Conference, Dec. 1978.

[12] X. Lin and N. Shroff, "The fundamental capacity-delay tradeoff in large mobile ad hoc networks," in Proc. Third Annual Mediterranean Ad Hoc Networking Workshop, Bodrum, Turkey, June 2004.

[13] X. Lin, G. Sharma, R. Mazumdar, and N. Shroff, "Degenerate delaycapacity trade-offs in ad hoc networks with brownian mobility," IEEE/ACM Trans. Networking, vol. 52, no. 6, pp. 2777-2784, June 2006.

[14] S. Toumpis and A. Goldsmith, "Large wireless networks under fading, mobility, and delay constraints," in Proc. IEEE INFOCOM 2004, Hong Kong, China, March 2004.

[15] A. E. Gamal, J. Mammen, B. Prabhakar, and D. Shah, "Optimal throughput-delay scaling in wireless networks - part i: the fluid model," IEEE Trans. Inform. Theory, vol. 52, no. 6, pp. 2568-2592, Jun. 2006.

[16] - -, "Optimal throughput-delay scaling in wireless networks - part ii: Constant-size packets," IEEE Trans. Inform. Theory, vol. 52, no. 11, pp. 5111-5116, Nov. 2006.

[17] G. Sharma, R. R. Mazumdar, and N. B. Shroff, "Delay and capacity trade-offs in mobile ad hoc networks: A global perspective," in Proc. IEEE INFOCOM 2006, Barcelona, Spain, April 2006.

[18] L. Ying, S. Yang, and R. Srikant, "Optimal delay-throughput tradeoffs in mobile ad hoc networks," IEEE Trans. Inform. Theory, vol. 54, no. 9, pp. 4119-4143, September 2008.

[19] T. Spyropoulos, K. Psounis, and C. S. Raghavendra, "Efficient routing in intermittently connected mobile networks: The multi-copy case," IEEE Trans. Networking, vol. 16, no. 1, pp. 77-90, Feb. 2008.

[20] A. Eryilmaz, A.Ozdaglar, and M. Medard, "On delay performance gains from network coding," in Proc. Conference on Information Sciences and Systems, Princeton, NJ, Mar. 2006.

[21] E. Ahmed, A. Eryilmaz, M. Medard, and A. Ozdaglar, "On the scaling law of network coding gains in wireless networks," in Proc. MILCOM 2007, Orlando, FL, Oct. 2007.

[22] M. Ghaderi, D. Towsley, and J. Kurose, "Reliability benefit of network coding," Tech. Report 07-08, Computer Science Department, University of Massachusetts Amherst, February 2007. [Online]. Available: http://www.ucalgary.ca/ mghaderi/docs/reliability.pdf

[23] J. Liu, D. Goeckel, and D. Towsley, "Throughput order of ad hoc networks employing network coding and broadcasting," in Proc. Milcom, Washington, DC, Oct. 2006.

[24] - - , "Bounds on the gain of network coding and broadcasting in wireless networks," in Proc. INFOCOM 2007, Anchorage, Alaska, May 2007.

[25] A. Keshavarz-Haddadt and R. Riedi, "Bounds on the benefit of network coding: Throughput and energy saving in wireless networks," in Proc. INFOCOM 2008, Phoenix, AZ, April 2008.
[26] M. Penrose, Random Geometric Graphs. Oxford: Oxford University Press, 2003.

[27] R. Meester and R. Roy, Continuum Percolation. Cambridge: Cambridge University Press, 1996.

[28] O. Dousse, M. Franceschetti, and P. Thiran, "Information theoretic bounds on the throughput scaling of wireless relay networks," in Proc. IEEE INFOCOM 2005, Miami, FL, Mar. 2005.

[29] S. Deb, M. Medard, and C. Choute, "Algebraic gossip: A network coding approach to optimal multiple rumor mongering," IEEE Trans. Inform. Theory, vol. 52, no. 6, pp. 2486-2507, June 2006.

[30] C. Fragouli, J. Widmer, and J.-Y. L. Boudec, "Efficient broadcasting using network coding," IEEE Trans. Networking, vol. 16, no. 2, pp. 450-463, April 2008.

[31] Y. Lin, B. Li, and B. Liang, "Stochastic analysis of network coding in epidemic routing," IEEE J. Sel. Areas Communications, vol. 26, no. 5, pp. 794-808, June 2008.

[32] S. Kulkarni and P. Viswanath, "A deternimistic approach to throughput scaling in wireless networks," IEEE Trans. Inform. Theory, vol. 50, no. 6, pp. 1041-1049, Jun. 2004.

[33] H. Kesten and V. Sidoravicius, "The spread of a rumor or infection in a moving population," Annals of Probability, vol. 33, no. 6, pp. 2402 2462, 2005.

[34] D. Aldous and J. Fill, "Reversible markov chains and random walks on graphs," Monograph in preparation. [Online]. Available: http://www.stat.berkeley.edu/ aldous/RWG/book.html

[35] M. Neely, E. Modiano, and C. E. Rohrs, "Dynamic power allocation and routing for time varying wireless networks," IEEE J. Sel. Areas Communications, vol. 23, no. 1, pp. 89-103, January 2005.

[36] M. J. Neely, "Dynamic power allocation and routing for satellite and wireless networks with time varying channels," PhD thesis, Massachusetts Institute of Technology, 2003.

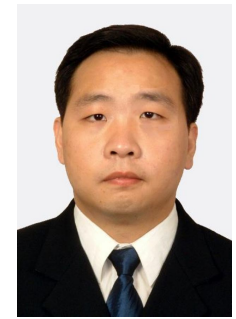

Chi Zhang (IEEE, S'06) received the B.E. and M.E. degrees in Electrical Engineering from Huazhong University of Science and Technology, Wuhan, China, in July 1999 and June 2002, respectively. He is now working towards his Ph.D. degree in the Department of Electrical and Computer Engineering at the University of Florida. His research interests include network security, wireless networking and mobile computing.

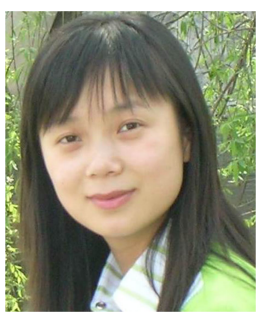

Xiaoyan Zhu received her BE degree in Information Engineering from Xidian University, Xian, China, in July 2000, and her ME degree in Information and Communications Engineering from Xidian University, Xian, China, in March 2004. She is now working towards her Ph.D. degree at Xidian University. Her research interests include wireless security and network coding.

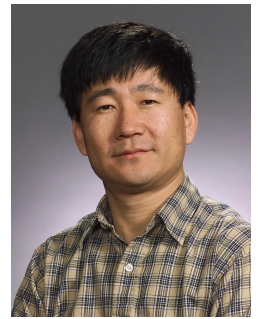

Yuguang "Michael" Fang (IEEE, M'97-SM'99. F'08) is a professor with University of Florida since 2005 and holds a University of Florida Research Foundation (UFRF) Professorship from 2006 to 2009. He received the NSF Career Award and ONR Young Investigator Award. He is the Editor-inChief for IEEE Wireless Communications and also serves/served on several editorial boards of technical journals including IEEE Transactions on Mobile Computing, IEEE Transactions on Communications, IEEE Transactions on Wireless Communications, IEEE Wireless Communications and ACM Wireless Networks. 